\title{
Low density interior in supercooled aqueous nanodroplets expels ions to the subsurface
}

\author{
Shahrazad M. A. Malek, ${ }^{\dagger}$ Ivan Saika-Voivod, ${ }^{\ddagger}$ and Styliani Consta*, \\ $\dagger$ Department of Physics and Physical Oceanography, Memorial University of \\ Newfoundland, Canada, A1B $3 X^{\eta}$ \\ $\ddagger$ Department of Physics and Physical Oceanography, Memorial University of \\ Newfoundland, Canada, A1B $3 X^{7}$ \\ IDepartment of Applied Mathematics, Western University, London, Ontario, Canada, \\ N6A $3 K K^{\prime}$ \\ $\S$ Department of Chemistry, The University of Western Ontario, London, Ontario, Canada \\ N6A $5 B 7$ \\ E-mail: sconstas@uwo.ca
}

\section{Abstract}

The interaction between water and ions within droplets plays a key role in the chemical reactivity of atmospheric and man-made aerosols. Here we report direct computational evidence that in supercooled aqueous nanodroplets a lower density core of tetrahedrally coordinated water expels the $\mathrm{Na}^{+}$ions to denser and more disordered subsurface. In contrast, at room temperature, the radial distribution of a single $\mathrm{Na}^{+}$ion in the droplet core is nearly uniform. We analyze the spatial distribution of a single ion in terms of a reference electrostatic model. The energy of the system in the analytical model is expressed as the sum of the electrostatic and surface energy of a deformable droplet. The model predicts that the ion is subject to a harmonic potential centered at the droplet's center of mass. We name this effect "electrostatic confinement". The model's predictions are consistent with the simulation findings for a single $\mathrm{Na}^{+}$ion at room temperature but not at supercooling. We anticipate this study to be the starting point for investigating the structure of supercooled electrosprayed droplets that are used to preserve the conformations of macromolecules originating from the bulk solution.

\section{Introduction}

The spatial distribution of ions in droplets plays a decisive role in chemical reactivity in atmospheric and man-made aerosols. Applications of the man-made aerosols relevant to this study include spray-based ionization methods used in native mass spectrometry analysis ${ }^{1}$ and use of droplets as micro- (nano-) reactors for accelerating chemical synthesis. ${ }^{2-4}$ Aerosol droplets in the lower atmosphere carry a small charge determined by at most a few excess ions whereas droplets in thunderclouds and electrosprays are highly charged. In this article we study the structure of supercooled aqueous mesoscopic clusters charged with a single and multiple ions. Hereafter, we will use the term nanodroplets for these mesoscopic clusters.

The structure and stability of supercooled clusters composed of ionic species and up to a few tens of water molecules has fascinated scientists over several decades. Experiments have detected abundance of certain "magic" cluster sizes associated with clathrate structures $^{5}$ and have studied their reactivity relevant to atmospheric chemistry. ${ }^{6}$ Many of the 
experiments have used Fourier transform ion cyclotron resonance (FT-ICR) mass spectrometry where dominant evaporative cooling of clusters over heating due to the absorption of black body radiation from the warmer walls of the apparatus $^{5,6}$ has been reported. In certain FTICR experiments the temperature of clusters composed of $50-70 \mathrm{H}_{2} \mathrm{O}$ molecules is estimated to be 130 K-150 K. Supercooled droplets also play a critical role as preservation environments of peptide conformations sprayed from the bulk solution using a variable-temperature electrospray ionization source coupled with ion mobility spectrometry and mass spectrometry techniques for analysis of the conformations. ${ }^{1}$

Most of the computational studies ${ }^{7-9,9-13,13-17,17-26}$ are for clusters at room or elevated temperature, while there are relatively a few computational studies ${ }^{12,14}$ of the supercooled clusters due to fact that they are notoriously challenging to be equilibrated. Voth and co-workers ${ }^{14}$ have investigated the location of a single $\mathrm{Na}^{+}$, $\mathrm{Cl}^{-}$and $\mathrm{H}_{3} \mathrm{O}^{+}$ion in clusters of $\mathrm{H}^{+}\left(\mathrm{H}_{2} \mathrm{O}\right)_{100}$, $\mathrm{Na}^{+}\left(\mathrm{H}_{2} \mathrm{O}\right)_{20}, \mathrm{Na}^{+}\left(\mathrm{H}_{2} \mathrm{O}\right)_{17}, \mathrm{Na}^{+}\left(\mathrm{H}_{2} \mathrm{O}\right)_{100}$ and $\mathrm{Cl}-\left(\mathrm{H}_{2} \mathrm{O}\right)_{17}$ in the temperature range of $100 \mathrm{~K}$ $450 \mathrm{~K}$. They found that below the freezing temperature in clusters of $100 \mathrm{H}_{2} \mathrm{O}$ molecules $\mathrm{H}_{3} \mathrm{O}^{+}$and $\mathrm{Na}^{+}$were excluded from the cluster interior and tend to reside within a few monolayers of the surface. Above the cluster melting point, both the $\mathrm{Na}^{+}$and $\mathrm{Cl}^{-}$ions tend to be found nearer to the center.

The spatial distribution of multiple ions in nanodroplets has been investigated less. Previously ${ }^{27,28}$ we have reported atomistic simulations of the location of multiple ions in aqueous nanodroplets with diameter $\approx 2 \mathrm{~nm}-16 \mathrm{~nm}$ at a temperature range of $300 \mathrm{~K}$ to $450 \mathrm{~K}$. We have found that in droplets comprising $\approx 1000 \mathrm{H}_{2} \mathrm{O}$ molecules, the radial ion distribution (measured from the droplet's center of mass) is almost uniform. As the droplet size increases, the distribution shows a distinct maximum in the outer droplet layers. The distribution dies off toward the droplet's center of mass (COM) by a slow almost exponential decay. Toward the droplet exterior, the decay is determined by the ion size and shape fluctuations. ${ }^{27}$ The solution of the non-linear Poisson-Boltzmann equation for a spherical geometry ${ }^{29}$ is used as a reference model to compare with the atomistic simulations. The distribution of multiple ions and biological molecules such as peptides at supercooling is still completely unknown.

Here we study the location of a single ion and multiple ions in relation to the solvent organization in supercooled aqueous clusters with sizes that vary from $100 \mathrm{H}_{2} \mathrm{O}$ molecules (corresponding to a diameter of $\approx 1.8 \mathrm{~nm}$ ) to 1100 (diameter $\approx 4.0 \mathrm{~nm}$ ). Our recent computer simulations ${ }^{30}$ for pristine supercooled water clusters have revealed an anomalous, inverted radial density profile emerging for $N \geq 200$ molecules at low temperature, in which a lowdensity core with relatively good tetrahedral ordering $^{31}$ is surrounded by a high density subsurface. These observations have some commonalities with studies of nucleation within droplets and thin films. ${ }^{32-36}$ We hypothesize that the low density water core in supercooled droplets will differentiate the ion distribution from that at room temperature. The hypothesis could not be explored in earlier research ${ }^{14}$ because the small cluster sizes (number of water molecules $\leq 100$ ) precluded the formation of the low density core.

In order to obtain insight into the forces that determine a single ion's location in a fluctuating droplet, we introduce a reference analytical model. To our knowledge, such a reference model is still missing from the literature. In the model the energy of a fluctuating droplet is the sum of the electrostatic energy and surface energy. It predicts that the ion is subject to a harmonic potential centered at the droplet's COM. For this reason we name this effect "electrostatic confinement" (EC). We present the conditions under which the electrostatic confinement is more evident.

\section{Theory of Electrostatic Con- finement}

Here, we present the key points of an analytical model that provides insight into the forces that determine the location of a single (macro)ion in a droplet. The details of the model are 
found in Sec. S1 in the SI. In the model we consider a charged dielectric droplet that may undergo shape fluctuations. The charge carrier is a single (macro)ion with bound charge (the macroion cannot ionize). The energy of the charged droplet is written as the sum of the electrostatic energy and surface energy.

After some algebra (details are found in Sec. S1 in SI), it is found that the energy related to the distance of the ion from the droplet's COM (denoted by $\Delta E_{1}$ ) is given by

$$
\Delta E_{1}(\|\mathbf{r}\|)=\frac{\varepsilon-1}{4 \pi \epsilon_{0} \varepsilon(\varepsilon+2)} \frac{Q^{2}}{R^{3}}\|\mathbf{r}\|^{2}
$$

where $Q, R$ and $\varepsilon$ are the charge of the ion, the droplet radius and the relative dielectric constant of the solvent, respectively, $\epsilon_{0}$ is the vacuum permittivity and $\|\mathbf{r}\|^{2}=X_{\mathrm{COM}}^{2}+Y_{\mathrm{COM}}^{2}+$ $Z_{\mathrm{COM}}^{2}$ (where $X_{\mathrm{COM}}, Y_{\mathrm{COM}}, Z_{\mathrm{COM}}$ are the coordinates of the droplet's COM). The energy (1) has the functional form of a harmonic potential. From this point we would refer to this effect as "electrostatic confinement" (EC). We introduce the spring constant $K(\varepsilon)$ where

$$
K(\varepsilon)=\frac{\varepsilon-1}{4 \pi \epsilon_{0} \varepsilon(\varepsilon+2)} \frac{Q^{2}}{R^{3}} .
$$

In Fig. 1 we plot the value of the spring constant as a function of the relative dielectric constant for a droplet comprising 1000 water molecules and an ion. The electrostatic energy has two limiting cases $\varepsilon=1$ and $\varepsilon=\infty$ when the electrostatic interaction of the ion with the droplet surface vanishes. In the former case the external and internal dielectric constants are equal and the droplet does not perturb the electric field of the ion. In the latter case the electrostatic field in localized in the vicinity of the ion and it is not affected by the droplet surface. As seen in the plot the maximum of the coefficient $K(\varepsilon)$ is attained at $\varepsilon=1+\sqrt{3} \approx 2.73$.

If the ion is localized in the center of a droplet the Gibbs-Boltzmann distribution of the ion positions is given by

$$
P\left(\|\mathbf{r}\|^{2}\right)=\frac{2}{\sqrt{\pi}}\left(\frac{K(\varepsilon)}{k_{B} T}\right)^{3 / 2}\|\mathbf{r}\| e^{-K(\varepsilon)\|\mathbf{r}\|^{2} / k_{B} T} .
$$

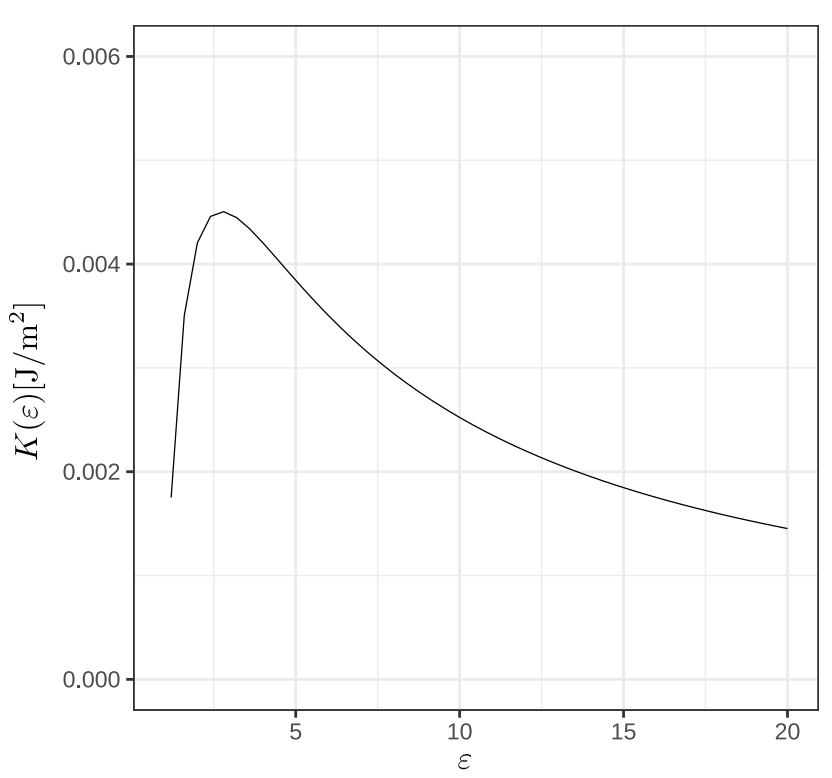

Figure 1: Magnitude of the spring constant as a function of the relative dielectric constant $\varepsilon$. The values correspond to an ion of charge $Q=$ $1 e$ in a droplet comprising 1000 water molecules and radius $19 \AA$. The value of the potential has a maximum at $\varepsilon \approx 1+\sqrt{3} \approx 2.73$.

The expectation value of the square of the distance of the ion from the center of mass is given by

$$
\left\langle\|\mathbf{r}\|^{2}\right\rangle=\frac{3}{2} \frac{k_{B} T}{K(\varepsilon)}
$$

The EC is more pronounced when the ion is localized at the center of the droplet, therefore we can write $\left\langle\|\mathbf{r}\|^{2}\right\rangle / R^{2} \ll 1$. Analyzing Eqs. (2) and (4) we conclude that the effect will be more pronounced at low temperature, high charge, small radius and intermediate values of the dielectric constant. Small droplet with high charge may undergo Rayleigh instability $^{37-40}$ leading to the "star" -shaped droplets ${ }^{41}$ (see Fig. S2 in SI). If this is an issue in observations we need to increase the droplet radius while keeping constant the value of the Rayleigh parameter $X \sim Q^{2} / R^{3}{ }^{42}$ To illustrate the EC we model high charges by creating models of charged cyclic peptides. DNA and RNA strands are other examples where the effect of the EC will be clearly observed.

Using the maximum value of the $K$ parameter from Fig. 1 we obtain the estimate of the 


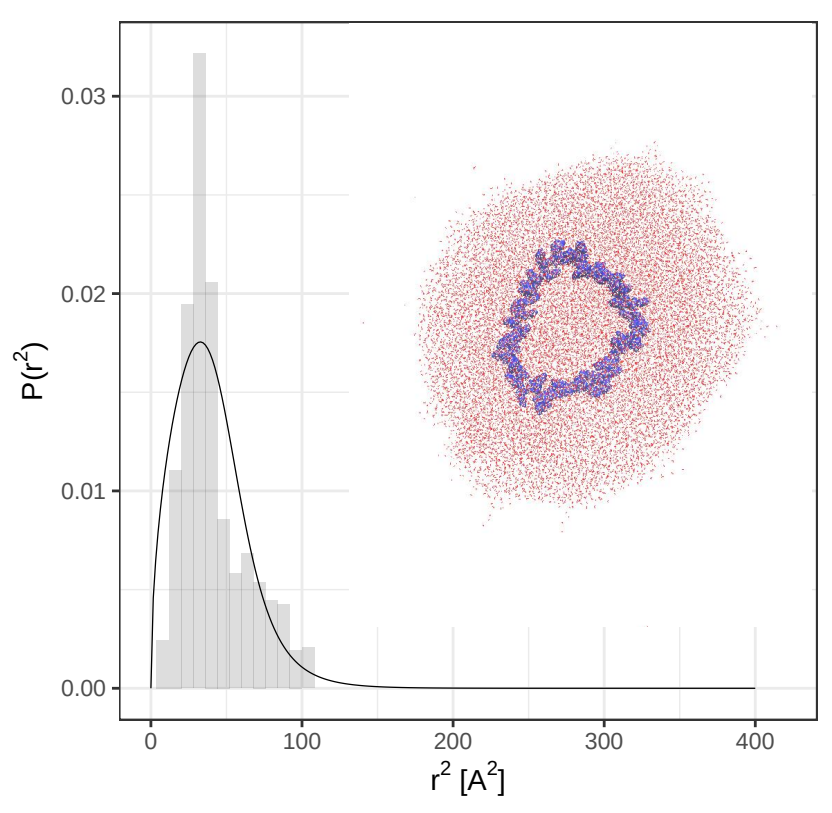

Figure 2: The distribution of the distances of the COM of the cyclic peptide (grey colored histogram) relative to the droplet's COM. The droplet radius is $3.8 \mathrm{~nm}$ and the charge is $8 \mathrm{e}^{+}$. The solid line is the gamma function fitted to the distribution using the maximum likelihood estimate (MLE). The inset shows a typical snapshot of the droplet composed of $8000 \mathrm{H}_{2} \mathrm{O}$ molecules (red colored) and the cyclic peptide (blue colored). minimal dimensions of the excursions of the ion from its equilibrium position at the center of the droplet $\sqrt{\left\langle\|\mathbf{r}\|^{2}\right\rangle} \geqslant 12 \AA$. Therefore, for a droplet comprising 1000 water molecules and a single charge $Q=1 e$ the geometric confining effects should be taken into consideration.

In Fig. 2 the distribution of the distance of the center of mass of the peptide relative to the COM of the droplet is plotted. The droplet's equimolecular radius is $3.8 \mathrm{~nm}$ and the charge is $8 \mathrm{e}^{+}$. The distribution tapers off before reaching the droplet surface. Sampling proves to be a challenge in such systems. The simulation time should be much longer than the time for a molecule to diffuse the width of the droplet $D t_{\text {sim }} \gg R^{2}$. Typical values of the diffusion coefficient $(D)$ are $\sim 10^{-9}\left[\frac{\mathrm{m}^{2}}{\mathrm{~s}}\right]$, hence the simulation time has to be in $10 \mathrm{~ns} 100 \mathrm{~ns}$ range at temperature $T=300 \mathrm{~K}$.

In summary, there is always a force on an ion toward the droplet COM. The electrostatic confinement effect is more pronounced for an ion with a charge of at least $\pm 3 e$ (where $e$ is the elementary positive charge) found in a droplet with a small radius. The radius size is equal or moderately larger than the radius at the Rayleigh limit. ${ }^{37-40}$ The Rayleigh limit is defined as the point where the electrostatic forces balance the surface forces. For a radius smaller than that at the Rayleigh limit, the droplet shows instability, which is manifested by "star"shapes.

\section{Models and Simulation Methods}

We simulate $\mathrm{Na}^{+}$ions in aqueous nanodroplets at $T=200 \mathrm{~K}, 260 \mathrm{~K}$, and $300 \mathrm{~K}$, representing the standard and supercooled conditions. The system sizes and length of simulations are shown in Table 1. The simulations are performed by molecular dynamics (MD) as implemented in GROMACS v4.6.1. ${ }^{43-46}$ The water molecules are modeled with the TIP4P/2005 (transferable intermolecular potential with four points) model. ${ }^{47}$ For $\mathrm{Na}^{+}$the OPLS-AA parameters are taken from Ref. ${ }^{48}$ Specifically, the ion has charge $+1 e$ (where $e$ is the elementary pos- 
Table 1: Simulation parameters. $N$ denotes the number of $\mathrm{H}_{2} \mathrm{O}$ molecules in the simulation box of dimension $L$. $N_{d}$ is the average number of the $\mathrm{H}_{2} \mathrm{O}$ molecules that form a connected drop, $\tau$ is the relaxation time and $t_{\text {run }}$ is the duration of the run. The "*" superscript in the temperature refers to simulations started with $\mathrm{Na}^{+}$ion(s) in or near the droplet center. $R_{e}$ denotes the equimolecular radius.

\begin{tabular}{|c|c|c|c|c|c|c|c|}
\hline $\begin{array}{c}N \\
\left(\mathrm{H}_{2} \mathrm{O}\right)\end{array}$ & $\begin{array}{c}L \\
(\mathrm{~nm})\end{array}$ & $\begin{array}{c}T \\
(\mathrm{~K})\end{array}$ & $N_{\mathrm{Na}}$ & $\begin{array}{c}t_{\mathrm{run}} \\
(\mu \mathrm{s})\end{array}$ & $N_{\mathrm{d}}$ & $\begin{array}{c}R_{e} \\
(\mathrm{~nm})\end{array}$ & $\begin{array}{c}\tau \\
(\mathrm{ns})\end{array}$ \\
\hline \hline \multirow{2}{*}{100} & 10 & 200 & 1 & 1.6 & 100 & 0.87 & 0.4 \\
& & 260 & 1 & 1.6 & 99.8 & & 0.8 \\
\hline \multirow{2}{*}{200} & 10 & 200 & 1 & 1.6 & 200 & 1.10 & 0.8 \\
& & 260 & 1 & 1.6 & 199.8 & & 0.8 \\
\hline \multirow{3}{*}{360} & \multirow{2}{*}{10} & 200 & 1 & 1.6 & 360 & 1.35 & 0.4 \\
& & $200^{*}$ & 1 & 0.44 & 360 & 1.35 & 0.4 \\
& & 300 & 1 & 1.6 & 359.3 & 1.35 & 0.2 \\
\hline \multirow{3}{*}{776} & \multirow{2}{*}{15} & 200 & 1 & 0.33 & 776 & 1.77 & 0.8 \\
& & $200^{*}$ & 1 & 0.46 & 776 & 1.77 & 0.8 \\
& & $200^{*}$ & 5 & 0.53 & 776 & 1.77 & 0.8 \\
\multirow{2}{*}{1100} & 20 & 200 & 1 & 0.77 & 773.9 & 1.75 & 0.4 \\
\hline & & $200^{*}$ & 8 & 0.32 & 1100 & 2.0 & 1.6 \\
& & 300 & 1 & 0.32 & 1095.1 & 1.97 & 0.8 \\
\hline
\end{tabular}

itive charge), and Lennard-Jones parameters $\epsilon=0.0115980 \mathrm{~kJ} / \mathrm{mol}$, and $\sigma=0.333045 \mathrm{~nm}$. In interacting with the $\mathrm{O}$ site of the water molecules the combining rules $\epsilon_{\mathrm{Na}, \mathrm{O}}=\sqrt{\epsilon_{\mathrm{Na}} \epsilon_{\mathrm{O}}}$ and $\sigma_{\mathrm{Na}, \mathrm{O}}=\sqrt{\sigma_{\mathrm{Na}} \sigma_{\mathrm{O}}}$ are used.

Each nanodroplet has been placed in a periodic cubic box of length $L$ (see Table 1 ). The box is large enough to avoid any interaction between the water droplet and its periodic images. The length of cutoff for interactions (Coulomb and Lennard-Jones) is at $L / 2$, which is much larger than the droplet's diameter in order to reproduce long range electrostatic interactions within the droplet. The simulations are carried out in the canonical ensemble - constant number of molecules $N$, volume $V$, and $T$. The temperature is controlled by using the NoséHoover thermostat with time constant 0.1 ps. The equations of motion are integrated with the leap-frog algorithm with a time step of 2 fs.

The simulations are initiated with a condensed pure water nanodroplet where the $\mathrm{Na}^{+}$ ion is placed at the surface for the majority of the single $\mathrm{Na}^{+}$runs, and in the center for two runs at $N=360$ and 776 at $T=200 \mathrm{~K}$. All runs with multiple $\mathrm{Na}^{+}$ions start with the ions near the droplet center of mass.

In Table 1 , the mean number of molecule, $N_{d}$, forming the connected cluster (i.e. those not in the vapor), and the relaxation time $\tau$, determined from the neighbor correlation function are shown. ${ }^{30}$ The values of $\tau$ provide an estimate for the relaxation time for simulations that include ions. In the temperature range where simulations are performed the solvent evaporation within the simulation box is negligible.

To ensure that the we sufficiently sample an equilibrated system after the addition of a single $\mathrm{Na}^{+}$ion at $200 \mathrm{~K}$, where the concern for equilibration is the highest, we run two simulations for each of $N=360$ and $N=776$ nanodroplets. In one set, we set the $\mathrm{Na}^{+}$ion at or near the centre of the droplet, quench the system through a conjugate-gradient energy minimization, and then proceed with an MD simulation. In the other, we initially place the ion on the surface. Equilibration time is estimated from the time it takes for the results of the simulations from the two different conditions to con- 
verge. For example, for $N=776$ after $400 \mathrm{ns,}$ the ion densities as a function of radial distance from the droplet COM $\rho_{\text {ion }}(r)$ converge for the two simulations. For $N=1100$, we assume that the equilibration time is longer by a factor of $\tau_{1100} / \tau_{776} \approx 2$. For multiple ions, initially distributed in the nanodroplet interior, we presume that the relaxation time is shorter and that the single ions simulations provide upper bounds on the relaxation times.

To help understand the features of $\rho_{\text {ion }}(r)$ in terms of the structure of pure water nanodroplets, we report a few other quantities as a function of radial distance from the pure water nanodroplet COM. These include the density of water $\rho(r)$, the density of water as determined from the Voronoi volumes associated with each water molecule $\rho_{v}(r)$, the tetrahedral order parameter $q_{T}(r)$ and the distance to the fifth nearest neighbor $\mathrm{O}$ of a given $\mathrm{O}$ atom $d_{5}$.

In our previous study, ${ }^{30}$ the Voronoi cells for all $\mathrm{O}$ sites were computed, while the $\mathrm{H}$ sites were ignored. Within each shell of radius $r$, the total volume $\mathcal{V}(r)$ of the Voronoi cells for O sites was found, and the same for the number of $\mathrm{O}$ sites in the shell, $\mathcal{N}(r)$. The average density as determined by the Voronoi cell volumes is defined as $\rho_{v}(r)=m\langle\mathcal{N}(r) / \mathcal{V}(r)\rangle$, where $\langle\cdots\rangle$ indicates an average over the configurations sampled in the simulations, and $m$ is the mass of a water molecule.

To characterize the local structures, we determine the tetrahedral order parameter, which is defined at the level of a single particle as, ${ }^{49}$

$$
q_{i}=1-\frac{3}{8} \sum_{j=1}^{3} \sum_{k=j+1}^{4}\left[\cos \psi_{j i k}+\frac{1}{3}\right]^{2}
$$

where $\psi_{j i k}$ is the angle between an oxygen atom $i$ and its nearest neighbor oxygen atoms $j$ and $k$ within a distance of $r_{c u t}=0.35 \mathrm{~nm}$. We define the radial function $q_{T}(r)$ as the average value of $q_{i}$ for all molecules within a spherical shell enclosed within $r \pm \Delta r / 2$, where $\Delta r=0.05 \mathrm{~nm}$. Similarly, we report results for $d_{5}(r)$, the average distance to the fifth $\mathrm{O}$ neighbor for $\mathrm{O}$ atoms located in the same spherical shell centered at $r$. Data for both $q_{T}(r)$ and $d_{5}(r)$ are taken from Ref. $^{31}$

\section{Results}

\section{Single $\mathrm{Na}^{+}$ion}

Figure 3 shows $\rho(r)$ and $\rho_{v}(r)$ for pure water nanodroplets of all sizes studied at high $(300 \mathrm{~K}$ for $N \geq 200,260 \mathrm{~K}$ for $N \leq 200$ ) and low $(200 \mathrm{~K})$ temperature $(T)$, with data taken from Ref. ${ }^{30}$ At high $T$, shown in Fig. $3(\mathrm{a}), \rho(r)$ is that of a typical liquid droplet, characterized by a flat (slowly decreasing) curve in the interior that decays sigmoidally to (near) zero over approximately an intermolecular distance at the liquid-vapor interface. The exceptions are the curves for $N \leq 200$, that at $260 \mathrm{~K}$ show some ordering or layering particularly near the surface. At low $T$, shown in Fig. 3 (b), there is significant layering for all nanodroplet sizes, and, as seen particularly well for the larger nanodroplets, an increase in density as $r$ increases towards the surface. Note that for $r<0.2 \mathrm{~nm}$, good statistics are difficult to obtain for all radial quantities, and results in this regime are quite noisy.

The undulations in $\rho(r)$ associated with layering make it difficult to characterize how the local density changes with $r$, and for this reason we plot $\rho_{v}(r)$. Fig. 3 (c) shows $\rho_{v}(r)$ monotonically decreasing (or flat) with $r$ for all nanodroplet sizes at high $T . \rho_{v}(r)$ is significantly smoother than $\rho(r)$, as it does not depend on the number density itself, but rather on the Voronoi volume surrounding each water molecule. An important feature of $\rho_{v}(r)$ is that it begins to decay to zero approximately $0.3 \mathrm{~nm}$, or an intermolecular distance, before $\rho(r)$; Voronoi volumes are very large, and Voronoibased density very low, for molecules on the surface. Molecules falling within the range where $\rho_{v}(r)$ is high (near bulk values) are completely surrounded by other water molecules and are not on the surface. Surface molecules can be identified as those located where $\rho_{v}(r)$ is small, and molecules in the subsurface as those located an intermolecular distance below the point at which $\rho_{v}(r)$ has decayed to near zero.

Fig. $3(\mathrm{~d})$ shows $\rho_{v}(r)$ for nanodroplets at low $T$. For $N \geq 200$, there is a significant increase in density in the subsurface layer. The density 


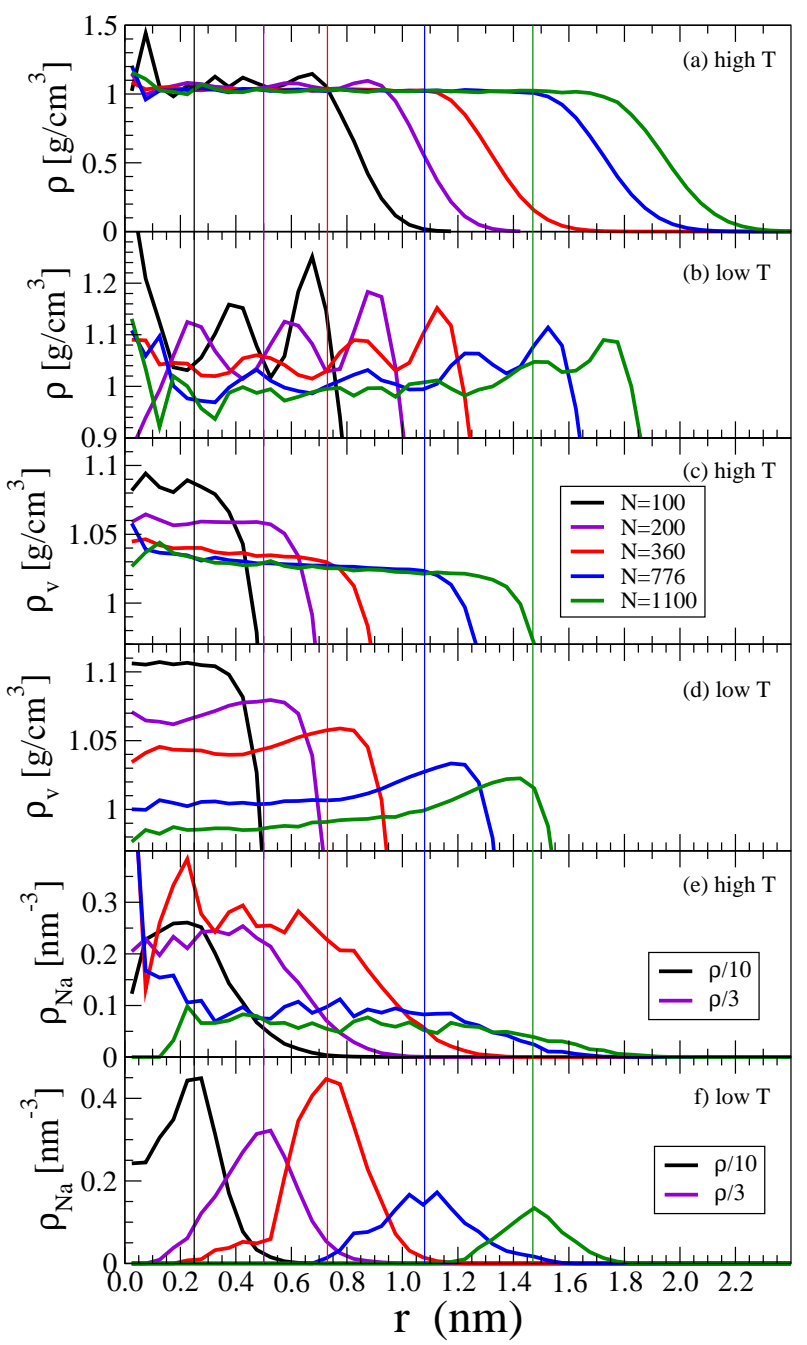

Figure 3: Pristine water nanodroplet structure and single $\mathrm{Na}^{+}$number density distributions for low temperature $(T=200 \mathrm{~K})$ and high temperatures $(T=260 \mathrm{~K}$ for $N \leq 200$ and $T=300 \mathrm{~K}$ for $N \geq 360$ ). Panels (a) and (b) show water density $\rho(r)$; panels (c) and (d) show water density based on molecular Voronoi volumes $\rho_{v}(r)$. Panels (e) and (f) show $\mathrm{Na}^{+}$number density $\rho_{\mathrm{Na}}(r)$, which for $N=100$ and $N=200$ have been reduced by a factor of 10 and 3, respectively. Note: to convert from molecule $/ \mathrm{nm}^{3}$ to $\mathrm{mol} / \mathrm{L}$, multiply by $10 / 6.022$.

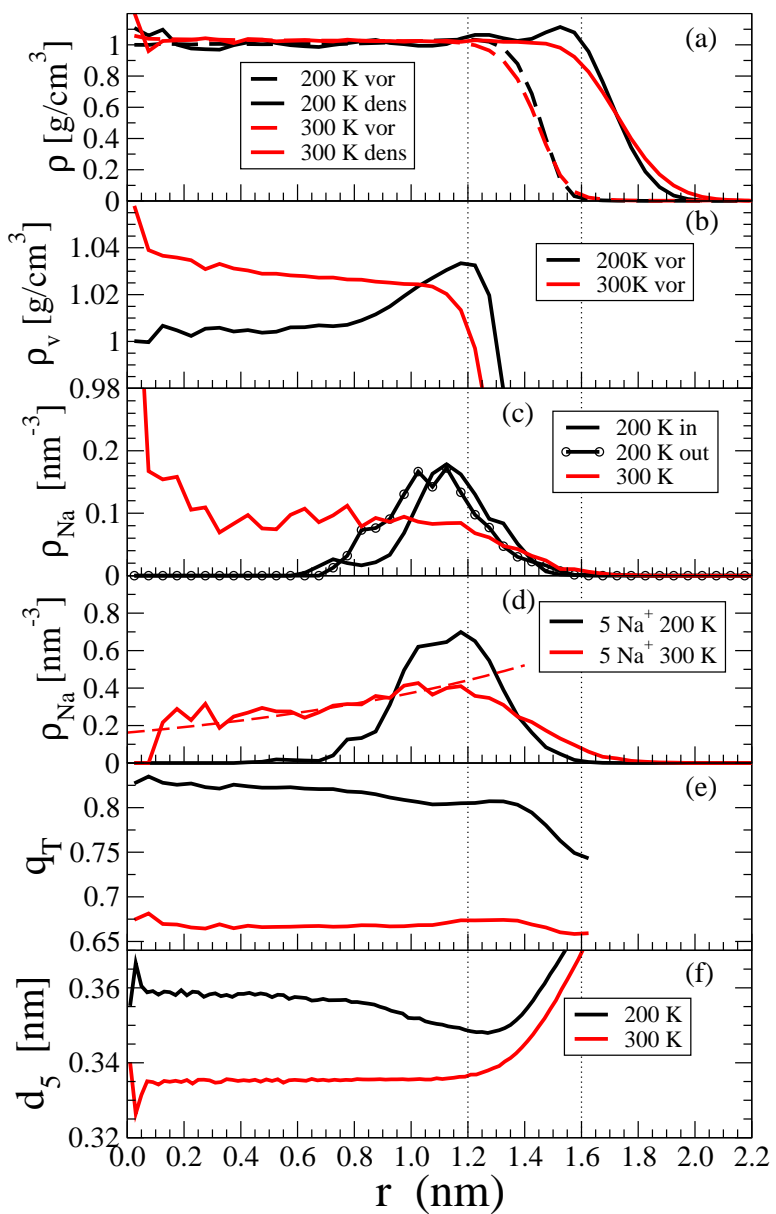

Figure 4: Structure of pristine water droplet, and single $\mathrm{Na}^{+}$and $5 \mathrm{Na}^{+}$distributions for $N=776$. Shown as functions of $r$ are (a) density $\rho ;($ b) density determined from Voronoi volumes $\rho_{v}$; (c) $\mathrm{Na}^{+}$density $\rho_{\mathrm{Na}}$ for a single ion, which for $200 \mathrm{~K}$ includes results from starting the ion at the centre (in) and at the surface (out); (d) $\mathrm{Na}^{+}$density $\rho_{\mathrm{Na}}$ when the nanodroplet contains 5 ions, dashed line indicating a fit to Eq. 6; (e) tetrahedral order parameter $q_{T}$; and (f) distance of the fifth $\mathrm{O}$ neighbor $d_{5}$. In all panels, black lines are for $200 \mathrm{~K}$, while red lines are for $300 \mathrm{~K}$. 
may well be higher for surface molecules, but $\rho_{v}(r)$ can not characterize this. This increased density at low $T$ appears to be a hallmark of cold water nanodroplets, and has not been reported for simple liquids to our knowledge. It is this heterogeneous environment in pure water that lends an interesting backdrop for studying ion distributions at low $T$.

In Fig. 3 (e), we plot $\rho_{N a}(r)$ at high $T$ for systems composed of a single $\mathrm{Na}^{+}$ion within a nanodroplet. Since the ion density is quite high for small nanodroplets, we divide $\rho_{N a}(r)$ by 10 and 3 for $N=100$ and 200, respectively. In all cases, the $\rho_{N a}(r)$ is approximately constant in the interior of the droplet, and begins to decay within the subsurface, and decays to zero significantly before $\rho(r)$ does. For a pure dielectric sphere at $T=0$, a point charge has lowest energy at the centre of a sphere. At finite $T$, there will be a finite width associated with the distribution of ion position. The convergence of the trajectories for $\mathrm{Na}^{+}$starting on the surface and the droplet's COM is shown in Fig. S3 in SI. At $T=300 \mathrm{~K}$ the $\mathrm{Na}^{+}$radial distribution is consistent with the EC effect. Because of the low charge, the geometric confinement effect dominates over the force toward the droplet's COM. For this reason, the $\mathrm{Na}^{+}$ radial distribution does not distinctly peak at the droplet's COM, but it appears to be almost uniform.

Fig. 3 (f) shows a dramatic difference in $\rho_{N a}(r)$ at low $T$. Rather than being centered at $r=0$, the peak of $\rho_{N a}(r)$ is located within $0.1 \mathrm{~nm}$ of the peak in $\rho_{v}(r)$ (for $N \geq 200$ ). Thus, we see that in a nanodroplet with a heterogeneous radial density, as determined by $\rho_{v}(r)$, the single $\mathrm{Na}^{+}$ion tends to reside in the highest density environment. This tendency is consistent with the fact that for constant $T$ and polarization factor (degree of dipole ordering), ${ }^{50}$ the dielectric constant increases with increasing density. While the surface layer is at very high density, as measured by $\rho(r)$, clearly the surface does not provide a good solvation environment.

For $N=100$ at low $T, \rho_{v}(r)$ is approximately constant for $r<0.3 \mathrm{~nm}$, and then decreases with increasing $r$. While a constant $\rho_{v}(r)$ for $r<0.3 \mathrm{~nm}$ suggests that $\rho_{N a}(r)$ should be uniform in this interior region, we see that $\rho_{N a}(r)$ is in fact peaked just below $0.3 \mathrm{~nm}$. We do see, however, that the peak in $\rho_{N a}(r)$ coincides with a local minimum in $\rho(r)$, suggesting that layering may play a significant role in determining where the $\mathrm{Na}$ ion resides in such small nanodroplets.

To probe the relationship between ion location and density a little further, we focus on the behaviour of the $N=776$ nanodroplet at $T=200 \mathrm{~K}$ and $T=300 \mathrm{~K}$ in Fig. 4: In panel (a) we replot $\rho(r)$ and $\rho_{v}(r)$ for pure water, showing their full range of values, confirming that the surface layer is approximately $0.3 \mathrm{~nm}$ thick; in panel (b) we show a close-up of $\rho_{v}(r)$ that contrasts the monotonic decrease at high $T$ with the monotonic increase towards a subsurface peak; in panel (c) we compare at low $T$ $\rho_{N a}(r)$ as obtained from starting the ion near the centre and starting near the surface - thus showing the degree of equilibration we achieve; panel (d) shows that the ion density when five $\mathrm{Na}^{+}$ions are present in the nanodroplet shifts at low $T$ to have a peak at approximately the same $r$ as for the single ion case; panel (e) shows the radial dependence of the tetrahedrality parameter, which has an approximately constant value at high $T$, while at low $T$ shows a significant decrease coinciding with the subsurface density peak; and panel (f) shows the radial dependence of $d_{5}$, a more indirect measure of the quality of the tetrahedral network, again showing a more disordered structure in the vicinity of the subsurface density peak at low $T$. The results shown in Fig. 4 confirm the idea that the ions, whether single or multiple, prefer the relatively disordered high density subsurface to the relatively ordered low density tetrahedral network of the core. We note that $q_{T}(r)$ begins to increase beyond a minimum located at $1.6 \mathrm{~nm}$, a location clearly in the surface layer where $d_{5}$ is quite high and $\rho_{v}$ is nearly zero. We thus cut off the $q_{T}(r)$ at this minimum, as the increase is not indicative of increased tetrahedral order. Single $\mathrm{Na}^{+}$number density radial distributions and measures of structure for low temperature $(T=200 \mathrm{~K})$ as those shown in Fig. 4 for select nanodroplet sizes $N=100,360,776$ and 1100 
are presented in Sec. S3 in SI. The same trend in the structure of the $\mathrm{H}_{2} \mathrm{O}$ and the location of the ions is found as for $N=776$. The cluster of $N=100$ does not show a tetrahedrally organized core. It is noted that this smaller cluster is subject to larger relative shape fluctuations than the larger droplets. Direct visual inspection revealed that the $\mathrm{Na}^{+}$lies one or two layers in from the cluster surface. Frequently the outer water molecules that participate in the immediate solvation of $\mathrm{Na}^{+}$lie parallel to the cluster surface. There is low tetrahedral structure close to the COM because the water molecules around the COM are adjacent to the first solvation shell of $\mathrm{Na}^{+}$, thus their organization is affected by the presence of the ion.

\section{Multiple $\mathrm{Na}^{+}$ions}

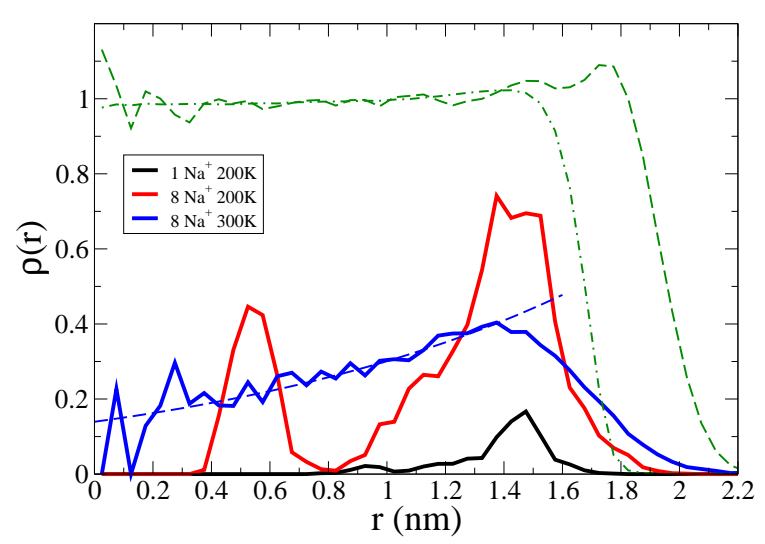

Figure 5: Single and $8 \mathrm{Na}^{+}, N=1100$. Data are for the last $35 \%$ of the time series for $200 \mathrm{~K}$. Dashed line is a fit of the $300 \mathrm{~K}$ data to Eq. 6 .

In Fig. 4 (d) and Fig. 5, the radial distributions of multiple ions are compared at $T=300 \mathrm{~K}$ and $200 \mathrm{~K}$ in droplets comprising 776 and $1100 \mathrm{H}_{2} \mathrm{O}$ molecules, respectively. At $T=300 \mathrm{~K}$ the distributions are almost uniform with an incipient broad maximum appearing in the outer layers of the droplet.

Solution $^{29}$ of the Non-linear PoissonBoltzmann (NPB) equation for a rigid spherical geometry suggests that toward the droplet interior the ion distribution will show an expo- nential decay

$$
n(r)=n(R) \exp \left[(r-R) / \lambda_{P B}\right]
$$

where $n$ is the ion number density, $R$ is the sphere radius (here taken to be equal to $R_{e}$ ) and $r$ is the distance from the droplet center. $\lambda_{P B}$ is given by

$$
\lambda_{\mathrm{PB}} \approx \frac{\epsilon k_{B} T}{\sigma q}
$$

where $k_{B}$ is Boltzmann constant, $T$ is temperature, $\epsilon$ is the permittivity, $q=m e$ is the charge of an ion ( $e$ is the elementary positive charge) and $\sigma$ is the surface charge density given by $\sigma=\frac{|Z| e}{4 \pi R^{2}}(|Z| e$ is the total droplet charge) In finding the surface charge density we assume that all the charge is in the surface. The larger the $\lambda_{P B}$ the slower the ion distribution decay. The simulated ion decay cannot be exactly as the theoretical prediction because of the droplet's shape fluctuations. The exponential decay will manifest more clearly in larger droplets at lower temperature. Obviously, the higher temperature will lead to a slower ion decay. For $776 \mathrm{H}_{2} \mathrm{O}$ molecules $-5 \mathrm{Na}^{+}$ions at $T=300 \mathrm{~K}$ the distribution decays (toward the droplet's COM) as an exponential function fitted by $0.42 \exp (-(1.14-x) / 1.2)$, where $\lambda_{P B} \approx$ $1.2 \mathrm{~nm}$. For $1100 \mathrm{H}_{2} \mathrm{O}$ the fitting function is $0.40 \exp (-(1.37-x) / 1.3)$, where $\lambda_{P B} \approx 1.3 \mathrm{~nm}$. Equation 7 with dielectric constant of water equal to 75 , yields $\lambda_{P B} \approx 0.8$. In droplets of up to a few thousands of water molecules the effect of shape fluctuations is significant, therefore, we interpret the value of $\lambda_{P B}$ only in a qualitative manner. The value of $\lambda_{P B}$ is comparable in size with the droplet radius, which indicates that the radial distribution function will show a very slow decay toward the COM, which is in qualitative agreement with the simulations. The ion distributions at $T=200 \mathrm{~K}$ shows a decay that cannot be analyzed using the NPB. The multiple ions are expelled from the drop's core and they show a maximum at the same location as the single ion. In droplets comprising $1100 \mathrm{H}_{2} \mathrm{O}$ molecules $-8 \mathrm{Na}^{+}$ions two peaks are observed at distance $0.52 \mathrm{~nm}$ and at $1.5 \mathrm{~nm}$. The lower intensity peak at $0.52 \mathrm{~nm}$ corresponds to one 
ion, which can exchange with the ions that give rise to the outer peak (at $\approx 1.5 \mathrm{~nm}$ ). Therefore, the appearance of two peaks does not indicate a metastable state.

\section{Conclusions}

We found that in supercooled aqueous droplets, a heterogeneous solvent structure leads to a different ion distribution relative to that at a room temperature. Specifically, we demonstrated that at supercooling a tetrahedral interior network can form, that expels a single or multiple $\mathrm{Na}^{+}$ions from the core region to the more disordered subsurface. The structure of the solvent has not been studied previously. A droplet of a supercooled $100 \mathrm{H}_{2} \mathrm{O}$-molecule droplet does not form a tetrahedral network in the center because the central $\mathrm{H}_{2} \mathrm{O}$ molecules participate in the immediate solvation of the $\mathrm{Na}^{+}$and the system is subject to large relative shape fluctuations. Voth et al. have studied the location of a single $\mathrm{Na}^{+}$in clusters with up to $100 \mathrm{H}_{2} \mathrm{O}$ molecules. ${ }^{14}$ Our results are in agreement with those of Voth et al. for the location of the $\mathrm{Na}^{+}$ion in cluster of $100 \mathrm{H}_{2} \mathrm{O}$ molecules. Because of the manner in which the $\mathrm{H}_{2} \mathrm{O}$ molecules are organized in the droplet's core at supercooling, the distribution of multiple ions cannot be explained by the predictions of the non-linear Poisson-Boltzmann equation.

We presented a reference model for justifying the location of a single-(macro)ion in a fluctuating droplet. The model considers the energy of a charged dielectric droplet as the sum of the electrostatic energy and surface energy. It is found that the ion is always subject to a harmonic potential centered at the droplet's center of mass. However, this electrostatic confinement is evident for certain droplet dimension and charge of the ion. The model predictions may deviate from the simulated ion distributions at supercooling, when the low density core may affect the location of the ions. The model can be extended further by including other factors such as the ion size and hydrophobic effects. The theory can serve as a reference model for comparing with the simulation data.
Evaporative cooling of droplets is a process that may be important in a droplet's lifetime in ionization methods used in native mass spectrometry. The implications of the distribution of multiple ions in supercooled droplets in a Rayleigh fission and and ion evaporation mechanism have to be examined. A conical deformation is a key component in the mechanism of ion emission. ${ }^{42} \mathrm{~A}$ related intriguing question is whether the formation of a conical deformation in the droplet that emits ions is hindered due to the low density core at supercooling. A future study on these questions will be relevant to atmospheric chemistry, native mass spectrometry and the physics of jets.

We anticipate our study to provide the starting point for investigating the structure of the low temperature droplets containing unstructured peptides and complexes of ions. These studies will assist in interpreting ion mobilitymass spectrometry data on the detection of the conformations of macromolecules in the bulk solution.

\section{Acknowledgments}

S.C. thanks Prof. D. Frenkel, Department of Chemistry, University of Cambridge, UK, and Dr. Anatoly Malevanets, The University of Western Ontario for discussions on the stability of charged systems. Professor D. Russell, Department of Chemistry, Texas A \& M University is thanked for pointing out the variable temperature ESI source and the role of cold droplets in transferring the protein conformations for MS analysis. I.S.-V. thanks the Departments of Applied Mathematics and Chemistry at Western University for sabbatical hosting. We acknowledge the financial support from Natural Sciences and Engineering Research Council (Canada). Computational resources were provided by ACENET and Compute Canada. 


\section{References}

(1) Raab, S. A.; El-Baba, T. J.; Woodall, D. W.; Liu, W.; Liu, Y.; Baird, Z.; Hales, D. A.; Laganowsky, A.; Russell, D. H.; Clemmer, D. E. Evidence for Many Unique Solution Structures for Chymotrypsin Inhibitor 2: A Thermodynamic Perspective Derived from vT-ESI-IMS-MS Measurements. J. Am. Chem. Soc. 2020, 142, 17372-17383.

(2) Bain, R. M.; Pulliam, C. J.; Cooks, R. G. Accelerated Hantzsch electrospray synthesis with temporal control of reaction intermediates. Chem. Sci. 2015, 6, 397-401.

(3) Ingram, A. J.; Boeser, C. L.; Zare, R. N. Going beyond electrospray: mass spectrometric studies of chemical reactions in and on liquids. Chem. Sci. 2016, 7, 39-55.

(4) Sahraeian, T.; Kulyk, D. S.; BaduTawiah, A. K. Droplet Imbibition Enables Non-Equilibrium Interfacial Reactions in Charged Microdroplets. Langmuir 2019, 35, 14451-14457.

(5) Lee, S.-W.; Freivogel, P.; Schindler, T.; Beauchamp, J. Freeze-dried biomolecules: FT-ICR studies of the specific solvation of functional groups and clathrate formation observed by the slow evaporation of water from hydrated peptides and model compounds in the gas phase. J. Am. Chem. Soc. 1998, 120, 11758-11765.

(6) Schindler, T.; Berg, C.; NiednerSchatteburg, G.; Bondybey, V. E. Heterogeneously catalyzed hydrolysis of chlorine nitrate: Fourier-transform ion cyclotron resonance investigations of stratospheric chemistry. J. Chem. Phys. 1996, 104, 3998-4004.

(7) Lu, D.; Singer, S. J. Ion solvation in model polar clusters. J. Chem. Phys. 1996, 105, 3700-3714.

(8) Gorlova, O.; DePalma, J. W.; Wolke, C. T.; Brathwaite, A.;
Odbadrakh, T. T.; Jordan, K. D.; McCoy, A. B.; Johnson, M. A. Characterization of the primary hydration shell of the hydroxide ion with $\mathrm{H} 2$ tagging vibrational spectroscopy of the $\mathrm{OH}^{-}-(\mathrm{H} 2 \mathrm{O})$ $\mathrm{n}=2,3$ and $\mathrm{OD}^{-}-(\mathrm{D} 2 \mathrm{O}) \mathrm{n}=2,3$ clusters. J. Chem. Phys. 2016, 145, 134304.

(9) Herce, D. H.; Perera, L.; Darden, T. A.; Sagui, C. Surface solvation for an ion in a water cluster. J. Chem. Phys. 2005, 122, 024513 .

(10) Perera, L.; Berkowitz, M. L. Ion solvation in water clusters. Zeitschrift für Physik D Atoms, Molecules and Clusters 1993, 26, 166-168.

(11) Thaunay, F.; Ohanessian, G.; Clavaguera, C. Dynamics of ions in a water drop using the AMOEBA polarizable force field. Chem. Phys. Lett. 2017, 671, 131137.

(12) Zhao, Y.; Li, H.; Zeng, X. C. Firstprinciples molecular dynamics simulation of atmospherically relevant anion solvation in supercooled water droplet. J. Am. Chem. Soc. 2013, 135, 15549-15558.

(13) Hagberg, D.; Brdarski, S.; Karlström, G. On the solvation of ions in small water droplets. The Journal of Physical Chemistry $B$ 2005, 109, 4111-4117.

(14) Burnham, C. J.; Petersen, M. K.; Day, T. J.; Iyengar, S. S.; Voth, G. A. The properties of ion-water clusters. II. Solvation structures of $\mathrm{Na}+, \mathrm{Cl}-$, and $\mathrm{H}+$ clusters as a function of temperature. $J$. Chem. Phys. 2006, 124, 024327.

(15) Makov, G.; Nitzan, A. Solvation and ionization near a dielectric surface. J. Phys. Chem. 1994, 98, 3459-3466.

(16) Fifen, J. J.; Agmon, N. Structure and spectroscopy of hydrated sodium ions at different temperatures and the cluster stability rules. J. Chem. Theory Comput. 2016, 12, 1656-1673. 
(17) Galib, M.; Baer, M.; Skinner, L.; Mundy, C.; Huthwelker, T.; Schenter, G.; Benmore, C.; Govind, N.; Fulton, J. L. Revisiting the hydration structure of aqueous Na+. J. Chem. Phys. 2017, 146, 084504.

(18) Perera, L.; Berkowitz, M. L. Structure and dynamics of Cl-(H2O) 20 clusters: The effect of the polarizability and the charge of the ion. J. Chem. Phys. 1992, 96, 82888294 .

(19) Perera, L.; Berkowitz, M. L. Erratum: Many-body effects in molecular dynamics simulations of $\mathrm{Na}+(\mathrm{H} 2 \mathrm{O}) \mathrm{n}$ and Cl(H2O) n clusters [J. Chem. Phys. 95, 1954 (1991)]. J. Chem. Phys. 1993, 99, 42364237.

(20) Perera, L.; Berkowitz, M. L. Many-body effects in molecular dynamics simulations of $\mathrm{Na}+(\mathrm{H} 2 \mathrm{O}) \mathrm{n}$ and $\mathrm{Cl}-(\mathrm{H} 2 \mathrm{O})$ n clusters. J. Chem. Phys. 1991, 95, 1954-1963.

(21) Caleman, C.; Hub, J. S.; van Maaren, P. J.; van der Spoel, D. Atomistic simulation of ion solvation in water explains surface preference of halides. Proc. Natl. Acad. Sci. U.S.A. 2011, 108, 6838-6842.

(22) Werhahn, J. C.; Akase, D.; Xantheas, S. S. Universal scaling of potential energy functions describing intermolecular interactions. II. The halide-water and alkali metal-water interactions. J. Chem. Phys. 2014, 141, 064118.

(23) Vaitheeswaran, S.; Thirumalai, D. Hydrophobic and ionic interactions in nanosized water droplets. J. Am. Chem. Soc. 2006, 128, 13490-13496.

(24) Harder, E.; Roux, B. On the origin of the electrostatic potential difference at a liquid-vacuum interface. J. Chem. Phys. 2008, 129, 12B613.

(25) Fracchia, F.; Del Frate, G.; Mancini, G.; Rocchia, W.; Barone, V. Force field parametrization of metal ions from statistical learning techniques. J. Chem. Theory Comput. 2018, 14, 255-273.

(26) Kwan, V.; Consta, S. Molecular Characterization of the Surface Excess Charge Layer in Droplets. J. Am. Soc. Mass Spectrom. 2021, 32, 33-45, PMID: 32597645.

(27) Kwan, V.; Malevanets, A.; Consta, S. Where do the ions reside in a highly charged droplet? J. Phys. Chem. A 2019, 123, 9298-9310.

(28) Kwan, V.; Consta, S. Bridging electrostatic properties between nanoscopic and microscopic highly charged droplets. Chem. Phys. Lett. 2020, 137238.

(29) Malevanets, A.; Consta, S. Variation of droplet acidity during evaporation. $J$. Chem. Phys. 2013, 138, 184312.

(30) Malek, S. M. A.; Poole, P. H.; SaikaVoivod, I. Thermodynamic and structural anomalies of water nanodroplets. Nat. Commun. 2018, 9, 2402.

(31) Malek, S. M. A.; Poole, P. H.; SaikaVoivod, I. Surface tension of supercooled water nanodroplets from computer simulations. J. Chem. Phys. 2019, 150, 234507.

(32) Johnston, J. C.; Molinero, V. Crystallization, melting, and structure of water nanoparticles at atmospherically relevant temperatures. J. Am. Chem. Soc. 2012, 134, 6650-6659.

(33) Nandi, P. K.; Burnham, C. J.; Futera, Z.; English, N. J. Ice-amorphization of supercooled water nanodroplets in no man's land. ACS Earth Space Chem. 2017, 1, 187-196.

(34) Li, T.; Donadio, D.; Galli, G. Ice nucleation at the nanoscale probes no man's land of water. Nat. Commun. 2013, 4, 16. 
(35) Haji-Akbari, A.; Debenedetti, P. G. Computational investigation of surface freezing in a molecular model of water. Proc. Natl. Acad. Sci. U.S.A. 2017, 114, 3316-3321.

(36) Hall, K. W.; Zhang, Z.; Burnham, C. J.; Guo, G.-J.; Carpendale, S.; English, N. J.; Kusalik, P. G. Does local structure bias how a crystal nucleus evolves? J. Phys. Chem. Lett. 2018, 9, 6991-6998.

(37) Rayleigh, L. XX. On the equilibrium of liquid conducting masses charged with electricity. Philos. Mag. 1882, 14, 184186.

(38) Peters, J. Rayleigh's electrified water drops. Eur. J. Phys. 1980, 1, 143.

(39) Hendricks, C.; Schneider, J. Stability of a conducting droplet under the influence of surface tension and electrostatic forces. Am. J. Phys 1963, 31, 450-453.

(40) Consta, S.; Malevanets, A. Disintegration mechanisms of charged nanodroplets: novel systems for applying methods of activated processes. Mol. Simul. 2015, 41, 73-85.

(41) Consta, S. Manifestation of Rayleigh instability in droplets containing multiply charged macroions. J. Phys. Chem. B 2010, 114, 5263-5268.

(42) Kwan, V.; O’Dwyer, R.; Laur, D.; Tan, J.; Consta, S. Relation between Ejection Mechanism and Ion Abundance in the Electric Double Layer of Droplets. $J$. Phys. Chem. A 2021, 0, 0-0.

(43) Berendsen, H. J. C.; van der Spoel, D.; van Druren, R. GROMACS: A messagepassing parallel molecular dynamics implementation. Comput. Phys. Commun. 1995, 91, 43.

(44) Lindahl, E.; Hess, B.; van der Spoel, D. GROMACS 3.0: A package for molecular simulation and trajectory analysis. J. Mol. Model. 2001, 7, 306.
(45) van der Spoel, D.; Lindahl, E.; Hess, B.; Groenhof, G.; Mark, A. E.; Berendsen, H. J. C. GROMACS: Fast, Flexible and Free. J. Comput. Chem. 2005, 26, 1701.

(46) Hess, B.; Kutzner, C.; van der Spoel, D.; Lindahl, E. GROMACS 4: Algorithms for highly efficient, load balanced, and scalable molecular simulation. J. Chem. Theory Comput. 2008, 4, 435.

(47) Abascal, J. L. F.; Vega, C. A general purpose model for the condensed phases of water: TIP4P/2005. J. Phys. Chem 2005, 123, 234505.

(48) Aqvist, J. Ion-water interaction potentials derived from free energy perturbation simulations. J. Phys. Chem. 1990, 94, 80218024.

(49) Chau, P. L.; Hardwick, A. J. Mol. Phys. 1998, 93, 511.

(50) Aragones, J. L.; MacDowell, L. G.; Vega, C. Dielectric Constant of Ices and Water: A Lesson about Water Interactions. J. Phys. Chem. A 2010, 115, $5745-5758$. 